\title{
Graphene for Dental Implant Applications
}

\author{
Akankshya Shradhanjali ${ }^{+1}$, Tasneem Bouzid ${ }^{+1}$, Alexander Sinitskiï and Jung Yul Lim² ${ }^{1,3 *}$ \\ ${ }^{1}$ Department of Mechanical and Materials Engineering, University of Nebraska-Lincoln, USA \\ ${ }^{2}$ Department of Chemistry, University of Nebraska-Lincoln, USA \\ ${ }^{3}$ Graduate School of Dentistry, Kyung Hee University, Korea
}

Submission: April 03, 2017; Published: April 13, 2017

*Corresponding author: Jung Yul Lim, University of Nebraska-Lincoln, W317.3 Nebraska Hall, Lincoln, NE, 68588-0526, USA, Tel: 1-402-472-2480; Fax: 1-402-472-1465; Email: jlim4@unl.edu

${ }^{+}$Contributed equally

Keywords: Graphene; Dental implant; Surface coating; Osteointegration; Functionalization

\section{Perspective}

Graphene is composed of a single layer of carbon atoms arranged as a hexagonal honeycomb lattice, exhibiting unique material characteristics such as mechanical, optical, thermal, electronic, and magnetic. Recently, biomedical researches have begun to exploit these unique properties of graphene and its derivative materials, opening a totally new research avenues in biomaterials, tissue engineering, and regenerative medicine $[1,2]$. In this perspective article, we will highlight recent studies that explore graphene-based materials for applications in dental implants and suggest perspective on potential future directions.

One crucial aspect in the fabrication of dental implants is the appropriate measure for preventing mechanical failure after implantation. Studies have examined the effect of graphenerelated carbon allotropes on implant mechanical properties. It was shown that silicatized titanium (Ti) plates had higher bond strength and good adhesion with carbon-graphite fiberreinforced (CGFR) polymer than the sandblasted counterpart [3]. Note, graphite, found in metamorphic rock, is composed of multiple layers of graphene. Also, the flexural strengths of CGFR polymers were maintained after hydrothermal cycling and the good adhesion property between graphite fibers and matrix was observed, suggesting promising applications of graphite fibers for prosthodontic field [4].

Another important challenge in the design and implementation of dental implants is the formation of oral biofilms and aggregation of primary etiological agents on the implant surface. To overcome this hindrance, researchers have attempted various implant surface coatings to achieve antibacterial properties. One recent study pursued graphene-based surface coating, i.e., coating of artificial acrylic teeth surface with non-toxic graphene/zinc oxide nanocomposite, and observed a substantial decrease in the deposition of the carcinogenic S. mutans bacteria and biofilm formation [5].

How to improve the osteointegration of dental implant body (screw) may be one of the most important issues that has to be improved. Numerous efforts have been made to enhance the osteointegration property by promoting the attachment, proliferation, and differentiation of bone forming cells on the implant surface. Recent studies have evidenced that graphene and related materials, e.g., graphene oxide (GO) and reduced graphene oxide (rGO), may provide excellent coating strategies for dental implants to improve osteointegration. Note, graphene denotes defect-free hexagonal carbon arrangement in a monolayer, which requires costly procedure for producing at a large scale. On the other hand, GO, highly oxidized graphene, can be made by a relatively simple, cost-effective method such as the phase exfoliation of graphite oxide. rGO is GO with subsequent and significant reduction in oxygen content.

In a recent study, a pristine graphene layer was fabricated on a nitinol (NiTi) shape memory alloy, which can be used for dental and orthopedic implants [6]. Such a graphene coating was found to significantly enhance the integrin-mediated focal adhesion and osteogenic differentiation of mesenchymal stem cells on the implant surface. Another study investigated the performance of GO-coated collagen scaffolds in osteoblastic cytocompatibility and alveolar bone healing of the tooth extraction socket [7]. GO scaffolds were found to support MC3T3-E1 cell proliferation in vitro. In rat subcutaneous implant testing, GO scaffolds could improve interconnected tissue structure, physical strength, enzyme resistance, protein adsorption, and cellular ingrowth relative to GO-uncoated control. Combined data suggest that GOcoated scaffolds would be beneficial for bone tissue engineering 
application. There is also a study to compare the biological activity of GO and rGO when applied for in vitro and in vivo osteogenesis tests [8]. In vitro data showed that MC3T3-E1 osteoblasts exhibited greater alkaline phosphatase activity on rGO-coated collagen scaffold than on GO-coated counterpart. Also, when these scaffolds were implanted on the back of rats, rGO-coated scaffolds induced increased tissue ingrowth relative to GO-coated and noncoated scaffolds, suggesting rGO may be even better candidate for orthopaedic and dental implant therapies.

It is of significance that for GO and rGO the existence of chemically reactive species $(-\mathrm{OH},-\mathrm{COOH}$, etc.) makes them excellent substrates for further functionalization. In a recent study, the surface of a Ti alloy (Ti13Nb13Zr) was modified with rGO loaded with osteogenic soluble factor (dexamethasone, Dex) through $\pi-\pi$ stacking on the graphitic domain of rGO [9]. The Dexloaded rGO coating of the dental implants induced significant enhancements in the growth, alkaline phosphatase activity, and bone-like mineral formation of MC3T3-E1 osteoblasts compared with non-Dex loaded samples. Another study attempted the incorporation of bone morphogenetic protein-2 (BMP-2), the growth factor known to stimulate cellular osteogenesis and bone formation, with GO [10]. BMP-2 was loaded on the GO-coated Ti substrate with the outermost coating layer of GO-COO-. The bone formation after eight weeks of implantation with the mouse calvarial defect model was significantly increased for the BMP-2 loaded GO substrate in comparison with $\mathrm{Ti}$ alone, Ti/GO, or Ti/ BMP-2 substrates, indicating that GO may play a role as an effective carrier for the controlled delivery of therapeutic proteins, such as BMP-2, to promote osteointegration of dental or orthopedic implants.

While studies highlighted above strongly suggest that graphene and related derivative materials would become high performance coating materials for dental and orthopaedic implants, it is still in the very early stage in both underlying science and practical applications with several issues to be explored. First, more systematic evaluations are required to test different combinations of base implant materials (Ti, CoCr, zirconium, tantalum, etc.) and graphene coating (graphene, GO, rGO). Such evaluations should include comprehensive material property characterizations including structural robustness of the graphene coating (defects, single or multi-layered) and coated surface characterizations (chemistry, roughness, hydrophilicity, surface energy). Then, osteogenic cytocompatibility should be tested in vitro utilizing various bone precursor cells (osteoblasts, stem cells from varying sources). For in vivo implantation tests, the implant-graphene combinations should be examined in regard to osteointegration (promotion of bone growth on the implant surface and the integration with the native tissue) and potential minimization capability of biofilm and debris formation.

Second, the potential capacity of graphene derivatives (GO, $\mathrm{rGO}$ ) to further incorporate bone formation stimulatory factors via chemical functionalization should be explored more. The examples noted above with dexamethasone and BMP-2 are good showcases, indicating a need for attempts using other osteogenic soluble factors, such as BMP-4/7, ascorbic acid, vitamin D, or molecular species capable of stimulating bone cell adhesion and osteogenic transcription. These studies on the delivery of therapeutic molecules via the functionalization of GO and rGO will request that the delivery carriers should enable the loading of sufficient biomolecular doses, enhanced adsorption, sustained release, and the retention of the biomolecular activity of the therapeutic molecules.

Third, it is true that very little has been revealed on why and how graphene-based implant coating will induce improved implant actions. In-depth molecular biology studies are highly required to determine molecular and cellular mechanisms of enhanced osteogenesis on the graphene materials. Since bone forming cells would see graphene coating as extracellular substrates to adhere on, the first and important target may be integrin-mediated focal adhesion, the formation of cytoskeletal structure to be anchored on the focal adhesion, and related signaling pathways. With all of these future studies being completed, the development of graphene coated implants with a higher degree of osteogenic cytocompatibility and minimum health hazard could be accomplished and the application of graphene materials for dental and orthopaedic tissue engineering and regenerative medicine purposes is expected to be blooming.

\section{Acknowledgement}

We appreciate the funding support from the NSF CAREER Award (1351570), Nebraska Research Initiative, and Layman New Directions Awards (all to J.Y. Lim).

\section{References}

1. Bouzid T, Sinitskii A, Lim JY (2016) Graphene platform for neural regenerative medicine. Neural Regen Res 11(6): 894-895.

2. Chen Y, Star A, Vidal S (2013) Sweet carbon nanostructures: carbohydrate conjugates with carbon nanotubes and graphene and their applications. Chem Soc Rev 42(11): 4532-4542.

3. Segerstrom S, Ruyter IE (2009) Adhesion properties in systems of laminated pigmented polymers, carbon-graphite fiber composite framework and titanium surfaces in implant suprastructures. Dent Mater 25(9): 1169-1177.

4. Segerstrom S, Ruyter IE (2009) Effect of thermal cycling on flexural properties of carbon-graphite fiber-reinforced polymers. Dent Mater 25(7): 845-851.

5. Kulshrestha S, Khan S, Meena R, Singh BR, Khan AU (2014) A graphene/ zinc oxide nanocomposite film protects dental implant surfaces against cariogenic Streptococcus mutans. Biofouling 30(10): 1281-1294.

6. Li J, Wang G, Geng H, Zhu H, Zhang M, et al. (2015) CVD growth of graphene on NiTi alloy for enhanced biological activity. ACS Appl Mater Interfaces 7(36): 19876-19881.

7. Nishida E, Miyaji H, Kato A, Takita H, Iwanaga T, et al. (2016) Graphene oxide scaffold accelerates cellular proliferative response and alveolar bone healing of tooth extraction socket. Int J Nanomedicine 11: 22652277.

8. Kanayama I, Miyaji H, Takita H, Nishida E, Tsuji M, et al. (2014) 
Comparative study of bioactivity of collagen scaffolds coated with graphene oxide and reduced graphene oxide. Int J Nanomedicine 9 3363-3373.

9. Jung HS, Lee T, Kwon IK, Kim HS, Hahn SK, et al. (2015) Surface modification of multipass caliber-rolled Ti alloy with dexamethasone- loaded graphene for dental applications. ACS Appl Mater Interfaces $7(18)$ : 9598-9607.

10. La WG, Park S, Yoon HH, Jeong GJ, Lee TJ, et al. (2013)Delivery of a therapeutic protein for bone regeneration from a substrate coated with graphene oxide. Small 9(23): 4051-4060.

\section{Your next submission with Juniper Publishers will reach you the below assets}

- Quality Editorial service

- Swift Peer Review

- Reprints availability

- E-prints Service

- Manuscript Podcast for convenient understanding

- Global attainment for your research

- Manuscript accessibility in different formats

(Pdf, E-pub, Full Text, Audio)

- Unceasing customer service

Track the below URL for one-step submission https://juniperpublishers.com/online-submission.php 